\title{
Prevalence of cardiovascular disease risk factors, and metabolic syndrome and its components in patients with psoriasis aged 30 to 49 years
}

\author{
Agnieszka B. Owczarczyk-Saczonek ${ }^{1}$, Roman J. Nowicki² \\ ${ }^{1}$ Department of Dermatology, Sexually Transmitted Diseases and Clinical Immunology, University of Warmia and Mazury, Olsztyn, \\ Poland \\ Head of the Department: Prof. Waldemar Placek MD, PhD \\ ${ }^{2}$ Department of Dermatology, Venereology and Allergology, Gdansk Medical University, Gdansk, Poland \\ Head of the Department: Prof. Roman Nowicki MD, PhD
}

Postep Derm Alergol 2015; XXXII (4): 290-295

DOI: $10.5114 /$ pdia.2014.40966

\begin{abstract}
Introduction: Some studies show that metabolic syndrome (MS) is more common in psoriatic patients.

Aim: To evaluate the prevalence of MS and its components as cardiovascular disease (CVD) risk factors in psoriatic patients compared to the general Polish population.

Material and methods: In 62 patients, aged 30 to 49 years with a mild to severe course of psoriasis, the features of MS have been assessed by IDF definition and compared to the results obtained in the NATPOL 2011 study.

Results: Analysis of CVD risk factors in patients with a severe course of psoriasis showed a correlation with waist circumference $(0.38, p<0.05)$, hypertension $(0.40, p<0.05)$ and a negative correlation with $\mathrm{HDL}(0.29, p<0.05)$. The prevalence of MS in psoriatic patients was $25.81 \%$, and for the control group $-21.02 \%(p>0.05)$, the mean HOMA-IR was 1.93 and $1.94(p>0.05)$, respectively. There were differences in the prevalence of abdominal obesity (53.6\% vs. $40.3 \%, p<0.05$ ). In lipid parameters, except for $\mathrm{HDL}$, the compared groups did not differ significantly (triglycerides, ApoA-I and B). Criteria for MS concerning blood pressure ( $>130 / 85 \mathrm{~mm} \mathrm{Hg}$ ) and hypertension were more frequent in men with psoriasis than in the control group $(38.2 \%$ vs. $11.1 \%, p<0.05)$.

Conclusions: Severe psoriasis is associated with a significantly higher prevalence of risk factors for CVD. The prevalence of MS, insulin resistance and lipid abnormalities in patients with psoriasis aged 30 to 49 years is similar to the general Polish population. Abdominal obesity is more common in psoriatic patients and hypertension in men with psoriasis.
\end{abstract}

Key words: psoriasis, metabolic syndrome, cardiovascular risk factors.

\section{Introduction}

Psoriasis affects 2-3\% of the European population and is now considered not only a skin disease, but also a systemic disease. In recent years, considerable progress has been made in clarification of the molecular mechanisms of the disease. Its association with metabolic syndrome and its components (abdominal obesity, insulin resistance, hypertension and dyslipidaemia) has been shown. Psoriasis may be a risk factor for the development of these diseases as well as an independent risk factor for myocardial infarction [1-5]. Th1 and Th17 lymphocytes and pro-inflammatory cytokines (such as TNF- $\alpha$, IL-1, IL-6, IL-17) play a decisive role in the patho- genesis of psoriasis and they also take part in forming atherosclerotic plaques and in development of insulin resistance [6-8]. The inflammation in psoriasis develops atherosclerotic and metabolic disorders, and vice versa. Therefore, the concept of 'psoriatic march' has been proposed to clarify the effect of severe psoriasis on the development of cardiovascular diseases [9]. Consequently, a dermatologist is not only responsible for treatment of skin lesions, but also for the diagnosis and monitoring of concomitant disorders. Early and appropriate treatment can prevent the development of associated metabolic disorders.

\footnotetext{
Address for correspondence: Agnieszka B. Owczarczyk-Saczonek MD, PhD, Department of Dermatology, Sexually Transmitted Diseases and Clinical Immunology, University of Warmia and Mazury, Aleja Wojska Polskiego 30, 10-595 Olsztyn, Poland, phone: +48 601 057 800, +48 8967866 70, fax: +48 8967866 75, e-mail: aganek@wp.pl Received: 7.08.2013, accepted: 2.01.2014.
} 


\section{Aim}

Numerous epidemiological studies show the link between psoriasis and cardiovascular diseases and metabolic disorders. The aim of this study was to evaluate the metabolic syndrome (MS) and its components (insulin resistance, atherogenic dyslipidaemia, abdominal obesity and hypertension) in patients with psoriasis aged 30 to 49 years compared to the adult Poles, who participated in the NATPOL 2011 study in the same age group. Additionally, an analysis has been made of the prevalence of risk factors for cardiovascular diseases depending on the severity of psoriasis.

\section{Material and methods}

The study included 62 consecutive patients with psoriasis aged 30 to 49 years treated at the out-patient clinic and the Department of Dermatology, Municipal Hospital in Olsztyn. The study group consisted of 28 (45.2\%) women and 34 (54.8\%) men. Among these, 24 patients had moderate and severe psoriasis (38.8\%) and 38 a mild form of the disease $(61.2 \%)$. Psoriatic patients with chronic and acute inflammatory diseases other than psoriasis, cancer, a history of cardiovascular complications and heart failure $(n=2)$ were excluded from the study.

The control group consisted of 861 people aged between 30 and 49 years, who were the representative, random sample of the Polish population: 402 women and 459 men with a negative history of psoriasis. These people were included in the NATPOL research project in 2011 conducted by a team from the Department of Hypertension and Diabetology, University Clinical Centre in Gdansk. The project involved the study of a representative group of 2411 Polish citizens aged between 18 and 78 years. The prevalence of hypertension and other risk factors for cardiovascular diseases among these patients were estimated. A detailed description of the sampling will be published in "Polish Cardiology".

The following parameters were estimated in all subjects: body mass index (BMI), waist circumference, blood pressure, according to the Polish Society of Hypertension Guidelines 2003. The dermatological examination assessed the severity of psoriasis - Psoriasis Area Severity Index (PASI), body surface area (BSA) and the index of the impact of skin diseases on the quality of life - Dermatology Life Quality Index (DLQI) [10]. Based on the above, the severity degree of psoriasis was measured, according to the new arrangements of the EUCOTT expert group (published in February 2011) [10, 11].

The Consensus divides psoriasis into:

- mild psoriasis, when the PASI, BSA and DLQI are $\leq 10$ points and then topical treatment is used;

- moderate to severe psoriasis, when the PASI or BSA is $>10$ points and DLQI is $>10$ points. Moderate to severe psoriasis is an indication for systemic therapy [11].
The patients had laboratory tests done under fasting conditions, $12 \mathrm{~h}$ after the last meal, evaluating the lipids ( $\mathrm{HDL}$, triglycerides, apolipoprotein A1 and B) and carbohydrates (glucose, insulin). Insulin resistance index HOMA-IR (Homeostasis Model Assessment-Insulin Resistance) was calculated [12]. To evaluate lipids, apolipoprotein A1 and B and ApoB/ApoA1 indices were estimated [13].

Metabolic syndrome was diagnosed in 2009 using the modified IDF (International Diabetes Federation) criteria [14], in which abdominal obesity (for Europeans: $\geq 94 \mathrm{~cm}$ men, women $\geq 80 \mathrm{~cm}$ ) is not a prerequisite for the diagnosis, but it is one of the 5 criteria. The diagnosis of metabolic syndrome is when 3 of 5 criteria are met (abdominal obesity, triglycerides level $\geq 150 \mathrm{mg} / \mathrm{dl}$ or treatment of hypertriglyceridaemia, $\mathrm{HDL}<40 \mathrm{mg} / \mathrm{dl}$ in men and $<50 \mathrm{mg} / \mathrm{dl}$ in women, blood glucose $\geq 100 \mathrm{mg} / \mathrm{dl}$ or pharmacotherapy of hyperglycaemia and blood pressure: systolic $\geq 130 \mathrm{~mm} \mathrm{Hg}$ and/or diastolic blood pressure $\geq 85 \mathrm{~mm} \mathrm{Hg}$ or antihypertensive drug treatment) [15].

\section{Statistical analysis}

Statistical analysis of the results was performed using the statistical software package "Statistica PL" v.10. Analysis of variance and correlation were used to verify the hypotheses.

\section{Results}

Cardiovascular disease risk assessment in patients with psoriasis depending on disease severity

Correlation analysis showed no correlation between the degree of severity and risk factors for cardiovascular diseases in cases of mild psoriasis. However, in patients with moderate and severe psoriasis, there is a correlation between the degrees of psoriasis severity and the following risk factors: waist circumference $0.38(p<0.05$ - a clear correlation and a negative correlation with HDL - 0.29 ( $p<0.05$ - a clear correlation) (Table 1).

\section{Evaluation of the prevalence of metabolic syndrome and its components}

The results showed no statistically significant difference between the NATPOL 2011 group and a group of patients with psoriasis in the incidence of MS. The prevalence of MS in patients with psoriasis was $25.8 \%$ and for the control group $21.0 \%$ ( $p>0.05)$.

There were no differences between the mean BMI and waist circumference in both groups, considering patients' gender. Mean BMI was $26.6 \mathrm{~kg} / \mathrm{m}^{2}$ in patients with psoriasis and $26.1 \mathrm{~kg} / \mathrm{m}^{2}$ for the control group ( $p>0.05$ ). Abdominal obesity was $88.4 \mathrm{~cm}$ and $90.1 \mathrm{~cm}$, respectively $(p>0.05)$. However, there were differences in the prevalence of abdominal obesity between the study group and the control group (53.6\% vs. $40.3 \%, p<0.05)$. 
Table 1. Comparison of average values of selected results between a sample group and a NATPOL 2011 group

\begin{tabular}{|c|c|c|c|c|c|c|}
\hline \multirow[t]{2}{*}{ Factor } & \multicolumn{3}{|c|}{ Sample group } & \multicolumn{3}{|c|}{ Control group - NATPOL 2011} \\
\hline & Total $(N=62)$ & Women $(N=28)$ & Men $(N=34)$ & Total $(N=861)$ & Women $(N=402)$ & Men $(N=459)$ \\
\hline BMI & $26.59 \pm 5.13$ & $25.88 \pm 5.50$ & $27.17 \pm 4.80$ & $26.09 \pm 4.67$ & $27.11 \pm 4.72$ & $27.20 \pm 4.36$ \\
\hline Waist circumference & $90.07 \pm 13.40$ & $88.28 \pm 16.90$ & $96.70 \pm 11.27$ & $88.82 \pm 14.47$ & $83.36 \pm 13.64$ & $93.32 \pm 13.73$ \\
\hline TG & $131.61 \pm 79.65$ & $108.43 \pm 71.68$ & $150.71 \pm 81.80$ & $127.26 \pm 97.15$ & $115.39 \pm 66.10$ & $156.7 \pm 118.71$ \\
\hline $\mathrm{HDL}$ & $54.82 \pm 18.01$ & $62.36 \pm 14.56$ & $48.62 \pm 18.40$ & $49.97 \pm 13.40$ & $60.68 \pm 19.94$ & $45.85 \pm 13.06$ \\
\hline Apo A 1 & $1.56 \pm 0.44$ & $1.64 \pm 0.43$ & $1.49 \pm 0.44$ & $1.56 \pm 0.37$ & $1.63 \pm 0.38$ & $1.51 \pm 0.35$ \\
\hline Apo B & $0.86 \pm 0.30$ & $0.79 \pm 0.43$ & $0.92 \pm 0.32$ & $0.91 \pm 0.24$ & $0.85 \pm 0.23$ & $0.97 \pm 0.25$ \\
\hline Index Apo B/Apo A I & $0.59 \pm 0.24$ & $0.49 \pm 0.15$ & $0.67 \pm 0.27$ & $0.61 \pm 0.21$ & $0.55 \pm 0.19$ & $0.67 \pm 0.21$ \\
\hline Glucose & $89.06 \pm 16.92$ & $86.16 \pm 17.55$ & $91.44 \pm 16.25$ & $90.98 \pm 16.34$ & $87.78 \pm 12.09$ & $93.75 \pm 17.73$ \\
\hline Insulin & $7.94 \pm 6.93$ & $7.33 \pm 7.08$ & $8.44 \pm 6.86$ & $8.24 \pm 6.57$ & $7.54 \pm 3.62$ & $8.85 \pm 7.52$ \\
\hline HOMA-IR & $1.93 \pm 2.13$ & $1.81 \pm 2.41$ & $2.03 \pm 1.90$ & $1.94 \pm 2.17$ & $2.30 \pm 1.93$ & $2.18 \pm 2.57$ \\
\hline Systolic pressure & $128.34 \pm 16.24$ & $122.46 \pm 12.40$ & $133.18 \pm 17.55$ & $126.60 \pm 16.55$ & $125.00 \pm 16.70$ & $126.12 \pm 16.08$ \\
\hline Diastolic pressure & $81.27 \pm 8.81$ & $78.46 \pm 9.14$ & $83.59 \pm 7.92$ & $80.56 \pm 10.77$ & $80.38 \pm 10.63$ & $80.72 \pm 10.88$ \\
\hline Hypertension, $n$ (\%) & $18(29 \%)$ & $5(17.8 \%)$ & 13 (38.2\%) & 207 (24\%) & $156(38.8 \%)$ & $51(11.1 \%)$ \\
\hline $\begin{array}{l}\text { Metabolic syndrome, } \\
n(\%)\end{array}$ & $16(25.8 \%)$ & $6(21.4 \%)$ & $10(29.4 \%)$ & $181(21.02 \%)$ & 46 (11.44\%) & 135 (29.41\%) \\
\hline Smoking, $n(\%)$ & $29(46.7 \%)$ & $9(32.1 \%)$ & $20(58.8 \%)$ & $250(29 \%)$ & $101(25.1 \%)$ & $149(32.4 \%)$ \\
\hline
\end{tabular}

There were no differences between the mean HOMA-IR and diabetes in both groups, even after considering patients' gender. The mean HOMA-IR was 1.93 in patients with psoriasis and 1.94 for the control group $(p>0.05)$.

Lipid analysis showed no difference between the mean triglycerides, ApoA1, ApoB, and ApoB/ApoA1 index, also considering the patients' gender. However, there was a significant increase in the HDL level in patients with psoriasis in comparison to the NATPOL 2011 control group.

Statistically significant differences were shown in the prevalence of MS criteria for blood pressure (> 130/85 $\mathrm{mm} \mathrm{Hg}$ ) in these groups considering patients' gender. In the study group, the prevalence of hypertension in patients is more than twice as high as in the NATPOL 2011 group and is $29 \%$ vs. $12.42 \%$ ( $p<0.01$ ), respectively. Moreover, in the group of men suffering from psoriasis, hypertension was observed more frequently than in men in the control group $(38.2 \%$ vs. $11.1 \%, p<0.05)$.

\section{Discussion}

Evaluation of risk factors for cardiovascular diseases in patients with psoriasis depending on disease severity

Recent studies suggest an increased risk of myocardial infarction in young patients with severe psoriasis com- pared with those without this disorder. In patients with psoriasis, the risk is about 20\% to $30 \%$ higher than in the control group [16]. Analysis of Gelfand et al. based on patients registered on the UK General Practice Research, showed that for a 30-year-old patient with severe psoriasis, the risk of heart attack is 3.10 and with mild psoriasis it is only 1.29. For a 60-year-old patient with both severe and mild course of psoriasis, the risk did not differ significantly from the general population, and was 1.36 and 1.08 respectively [17]. Similar results were obtained by Mehta et al., evaluating a relative risk of death from cardiovascular diseases, in the same group of patients. For 40-year-old patients with severe psoriasis it was 2.69, and for 60-year-old patients - 1.92 [11]. The criterion for severe psoriasis in both studies was the use of systemic therapy, not the EuCCOT classification system.

In our study, two of patients with a history of cardiovascular complications were excluded from the study. It could have had a very little influence on the observed correlation between psoriasis and risk factors for cardiovascular diseases.

\section{Occurrence of metabolic syndrome in patients with psoriasis}

Numerous epidemiological studies show that people with psoriasis are twice as likely to have MS compared to a healthy population, especially those with severe psoriasis [3, 18-20]. However, these are population-based 
studies of all adults and they do not take age into account. However, a study by Gisondi et al. showed that MS coexists inpatients with psoriasis in 30.1\% compared to patients with other dermatological diseases $-20.6 \%$, but over the age of 40 [21]. There are also conflicting reports $[22,23]$. Korean studies have confirmed this correlation, and people with psoriasis who had metabolic syndrome were much older (mean age $=53.3$ years) compared to patients without MS (mean age $=39.5$ years) [24]. Similar results were obtained by Pereira et al. when examining psoriasis patients aged 35-44years [25].

Many studies have confirmed the association between obesity and psoriasis and there is even a theory that obesity may be a risk factor for its occurrence [26, 27]. An increase in BMI [28-30] slightly increases the risk, but obesity $\left(\mathrm{BMI}>29 \mathrm{~kg} / \mathrm{m}^{2}\right)$ can double the risk of psoriasis [19, 27, 28]. Moreover, weight loss may improve psoriasis [29, 30], treatment response [31]. A large cohort study conducted among 78626 nurses in the United States, with a 14-year follow-up and BMI updated every 2 years, has shown that increased adiposity and weight gain are strong risk factors for psoriasis [19]. Similar results were obtained in a study on patients registered in the UK General Practice Research - 4,000 patients with psoriasis were involved. In patients with severe psoriasis odds ratio $(\mathrm{OR})$ was 1.8 and with mild psoriasis $1.3[1,26]$. A recent study conducted by Armstrong et al. (published in 2012) shows that the OR for obesity in patients with psoriasis was 1.66 compared to patients without psoriasis. Depending on the severity of the disease, it turned out that for patients with mild psoriasis it was 1.46 and with severe psoriasis -2.23 [32].

Our own research showed no statistically significant differences between the mean BMI but it confirmed that the prevalence of abdominal obesity increased in patients with psoriasis.

As early as in 1967, in studies conducted by Lynch, it was found that $29 \%$ of patients with psoriasis had abnormal blood glucose levels [33]. Polish studies have confirmed this as well in symptomatic psoriasis, although with resolution of clinical symptoms after treatment, serum glucose level stended to normalise [34, 35]. Studies conducted by Neimann et al. confirm the coexistence of diabetes in patients with severe psoriasis in $7.1 \%$ and with mild psoriasis in $4.4 \%$ compared to the control group (3.3\%) [36]. A weak but statistically significant association between the severity of psoriasis (PASI) and markers of insulin resistance (insulin, resistin levels) was observed in Boehencke studies [2, 9].

Our own research found no correlation between HOMA-IR and the severity of psoriasis. There was no difference in the prevalence of insulin resistance and diabetes between the patients and the NATPOL 2011 group.

Our results differ from numerous reports in the world literature. In Cohen et al.'s study evaluating the associations of diabetes in 340 patients with psoriasis, a fre- quent coexistence of these diseases was confirmed in the group. However, in patients below 35 years of age and between 35 and 50 years of age, the association is not statistically significant [3]. Reynosovon Drateln et al. also found no differences in insulin secretion and insulin resistance in patients with psoriasis compared to the control group.

However, they observed a correlation between psoriasis duration and insulin sensitivity [37]. In Polish studies, Janusz et al. showed slightly higher levels of insulin and HOMA-IR in psoriasis patients compared to the control group, but at the limit of statistical significance. There was found no correlation between these parameters and the disease duration either [38].

In own research, the age of the patients is an important factor influencing the results. This is particularly important in women. The balance of sex hormones plays a role in the development of insulin resistance and therefore the phenomenon is more common during menopause. The average age of Polish women at the time of menopause is $49 \pm 3.5$ years, which means that the health problems associated with menopause in Poland can be observed in women over 50 [39, 40]. Before experiencing menstrual disorders, there is a decrease in the level of growth hormone $\mathrm{GH}$ and insulin-like growth factor (IGF-1). The IGF-1 increases the uptake of glucose and amino acids through different tissues and stimulates glycogen synthesis in the liver. Therefore, a reduction in its level during menopause can have an influence on the creation of insulin resistance and the development of diabetes. Oestrogens deficiency during menopause also promotes the development of obesity, hypertension and the development of a full-blown MS [40, 41].

Numerous reports in the literature describe the frequent coexistence of lipid abnormalities in patients with psoriasis and psoriatic arthritis [1, 33, 42-44]. Moreover, the study conducted by Rocha-Pereira and by Polish researchers Toruniowa and Pietrzak confirm that lipid abnormalities depend on the severity of psoriasis [45-47].

Our own research showed no difference in the level of triglycerides, apolipoprotein A1, B and ApoB/ApoA1 index between the group of patients with psoriasis and the control group. However, an interesting observation is an elevated HDL levels demonstrated in patients with psoriasis.

Similar results were obtained by Mallbris et al. in patients with mild psoriasis. They had only a slight increase in the level of cholesterol and lipoproteins and mean HDL was even higher than in the control group [43]. The research by Pereira et al., conducted on patients with psoriasis aged 35-44, showed no statistically significant differences in lipid profile and increased incidence of MS compared to the control group [45].

The association between hypertension and psoriasis was first noted by Preece in 1977 [48]. The research conducted in 2010 by Cohen et al. confirmed that hypertension more frequently coexists in patients with psoriasis 
compared to healthy people (38\% vs. $29.1 \%$ ), but a significant association was observed in women over 40 and men over 30 [49]. Moreover, the research by Neimann et al. showed the relationship between this disorder and the severity of psoriasis: in patients with severe psoriasis $-20 \%$, and mild $-14.7 \%$ [34]. However, there are discrepancies in data which have not confirmed such an association $[21,50]$.

Our results showed that the prevalence of hypertension in men is twice as likely as in the NATPOL 2011 control group.

Our research has confirmed that severe psoriasis is associated with a higher prevalence of risk factors for cardiovascular diseases. The correlations of risk factors (abdominal obesity, lower $\mathrm{HDL}$ ) were found only in patients with severe and moderate psoriasis, but not in patients with mild psoriasis. The sample group of patients with psoriasis has comprised young people aged 30-49 years in relation to a large reference population participating in the NATPOL 2011 study. A new thing is classification criteria for psoriasis, according to the guidelines of the EuCCOT expert group. No such analyses are available in the Polish literature.

\section{Conclusions}

Severe psoriasis predisposes patients to the development of cardiovascular diseases. The prevalence of MS, insulin resistance and lipid abnormalities in patients with psoriasis aged 30 to 49 years is similar to that of the general adult population of Poland. Abdominal obesity is more common in patients with psoriasis. Hypertension is more common in men with psoriasis.

\section{Acknowledgments}

We thank Tomasz Zdrojewski MD, PhD, Professor Bogdan Wyrzykowski MD, PhD, Piotr Bandosz MD, PhD, Marcin Rutkowski MD, PhD of the Department of Hypertension and Diabetology, Medical University in Gdansk, for sharing their data from the NATPOL 2011 Survey.

\section{Conflict of interest}

The authors declare no conflict of interest.

\section{References}

1. Azfar RS, Gelfand JM. Psoriasis and metabolic disease: epidemiology and pathophysiology. Curr Opin Rheumatol 2008; 20: 416-22.

2. Boehncke S, Thaci D, Beschmann H, et al. Psoriasis patients show signs of insulin resistance. Br J Dermatol 2007; 157: 1249-51.

3. Cohen AD, Gilutz H, Henkin Y, et al. Psoriasis and the metabolic syndrome. Acta Dermatol Venereol 2007; 87: 506-9.

4. Gottlieb AB, Dann F, Menter A. Psoriasis and metabolic syndrome. J Drugs Dermatol 2011; 7: 563-72.
5. Perk J, De Backer G, Gohlke H, et al. European Guidelines on cardiovascular disease prevention in clinical practice (version 2012) The Fifth Joint Task Force of the European Society of Cardiology and Other Societies on Cardiovascular Disease Prevention in Clinical Practice (constituted by representatives of nine societies and by invited experts) Developed with the special contribution of the European Association for Cardiovascular Prevention \& Rehabilitation (EACPR). Eur Heart J 2012; 33: 1635-701.

6. Späh F. Inflammation in atherosclerosis and psoriasis: common pathogenic mechanisms and the potential for an integrated treatment approach. Br J Dermatol 2008; 159: 10-7.

7. Mehta NN, Azfar RS, Shin DB, et al. Patients with severe psoriasis are at increased risk of cardiovascular mortality: cohort study using the General Practice Research Database. Eur Heart J 2010; 31: 1000-6.

8. Shabgah AG, Fattahi E, Shahneh FZ. Interleukin-17 in human inflammatory diseases. Postep Derm Alergol 2014; 31: 256-61.

9. Boehncke WH, Boehncke S, Tobin AM, et al. The "psoriatic march": a concept of how severe psoriasis may drive cardiovascular comorbidity. Exp Dermatol 2011; 20: 303-7.

10. Szepietowski J, Salomon J, Finlay AY, et al. Dermatology Life Quality Index (DLQI): Polish version [Polish]. Dermatol Klin 2004; 6: 63-70.

11. Mrowietz U, Kragballe K, Reich K, et al. Definition of treatment goals for moderate to severe psoriasis: a European consensus. Arch Dermatol Res 2011; 301: 1-10.

12. Szurkowska M, Szafraniec K, Gilis-Januszewska A, et al. Insulin resistance indices in population-based study and their predictive value in defining metabolic syndrome [Polish]. Przegl Epidemiol 2005; 59: 743-51.

13. Brookes $L$. The apo B/A-I ratio - a stronger predictor of cardiovascular events than LDL, HDL, or total cholesterol, triglycerides, or lipid ratios. Medscape Cardiology 2006. Available at: http://www.medscape.com/viewarticle/541799

14. Wyrzykowski B. Definition of metabolic syndrome. In: Metabolic syndrome in clinical practice [Polish]. Wyrzykowski B (ed.). Via Medica, Gdansk 2010; 114-38.

15. Alberti KGMM, Eckel RH, Grundy SM, et al. Harmonizing the metabolic syndrome. Circulation 2009; 120: 1640-5.

16. Wakkee M, Nijsten T. Comorbidities in dermatology. Dermatol Clin 2009; 27: 137-47.

17. Gelfand JM, Neimann AL, Shin DB, et al. Risk of myocardial infarction in patients with psoriasis. J Am Med Assoc 2006; 296: 1735-41.

18. Love TJ, Quresi AA, Karlson EW, et al. Prevalence of the metabolic syndrome in psoriasis. Arch Dermatol 2011; 147: 419-24.

19. Setty AR, Curhan G, Choi HK. Obesity, waist circumference, weight change, and the risk of psoriasis in women: Nurses' Health Study II. Arch Intern Med 2007; 167: 1670-5.

20. Sommer DM, Jenisch S, Suchan M, et al. Increased prevalence of the metabolic syndrome in patients with moderate to severe psoriasis. Arch Dermatol Res 2006; 298: 321-8.

21. Gisondi P, Tessari G, Conti A, et al. Prevalence of metabolic syndrome in patients with psoriasis: a hospital-based case-control study. Br J Dermatol 2007; 157: 68-73.

22. Takahashi H, Tsuji H, Takahashi I, et al. Prevalence of obesity in Japanese psoriasis patients: adiposity is correlated with severity of psoriasis. J Dermatol Sci 2009; 55: 74-6.

23. Kutlu S, Ekmeci TR, Koslu A, et al. Prevalence of metabolic syndrome in patients with psoriasis. Indian J Dermatol Venereol Leprol 2011; 77: 193-4.

24. Gun-Wook K, Hyun P, Hoon-Soo K, et al. Analysis of cardiovascular risk factors and metabolic syndrome in Korean patients with psoriasis. Ann Dermatol 2012; 24: 11-5. 
25. Pereira RR, Amladi ST, Varthakavi PK. A study of the prevalence of diabetes, insulin resistance, lipid abnormalites and cardiovascular risk factors in patients with chronic plaque psoriasis. Indian J Dermatol 2011; 56: 520-6.

26. Bremmer S, van Voorhees AS, Hsu S, et al. Obesity and psoriasis: from the Medical Board of the National Psoriasis Foundation. J Am Acad Dermatol 2010; 63: 1058-69.

27. Baran A, Flisiak I, Jaroszewicz J, Świderska M. Effect of psoriasis activity on serum adiponectin and leptin levels. Postep Derm Alergol 2015; 32: 101-6.

28. Herron MD, Hinckley M, Hoffman MS, et al. Impact of obesity and smoking on psoriasis presentation and management. Arch Dermatol 2005; 141: 1527-34.

29. Gottlieb AB, Dann F. Comorbidities in patients with psoriasis. Am J Med 2009; 122: 1150-9.

30. Hamminga EA, van der Lely AJ, Neumann HA, et al. Chronic inflammation in psoriasis and obesity: implications for therapy. Med Hypotheses 2006; 67: 768-73.

31. Gisondi P, Del Giglio M, Di Francesco V, et al. Weight loss improves the response of obese patients with moderate-to-severe chronic plaque psoriasis to low-dose cyclosporine therapy: a randomized, controlled, investigator-blinded clinical trial. Am J Clin Nutr 2008; 88: 1242-7.

32. Armstrong AW, Harskamp CT, Armstrong EJ. The association between psoriasis and obesity: a systematic review and meta-analysis of observational studies. Nutr Diabetes 2012; 2: e54.

33. Tobin AM, Veale DJ, Fitzgerald O, et al. Cardiovascular disease and risk factors in patients with psoriasis and psoriatic arthritis. J Rheumatol 2010; 37: 1386-94.

34. Grzybowski G, Fąfara I, Żaba R, et al. Evaluation of glucose, insulin, C-peptide uric acid serum levels in patients with psoriasis [Polish]. Postep Derm Alergol 2002; 19: 46-51.

35. Seneczko F, Grzybowski G, Kaszuba A, et al. Carbohydrate metabolism in psoriasis [Polish]. Postep Derm Alergol 1997; 14: 337-43.

36. Neimann AL, Shin DB, Wang X, et al. Prevalence of cardiovascular risk factors in patient of psoriasis. J Am Acad Dermatol 2006; 55: 829-35.

37. Reynoso-von Drateln C, Martinez-Abundis E, Balcazar-Munoz BR, et al. Lipid profile, insulin secretion, and insulin sensitivity in psoriasis. J Am Acad Dermatol 2003; 48: 882-5.

38. Janusz I, Lewandowski K, Lukamowicz J, et al. Insulin resistance and adiponectin levels in psoriasis patients [Polish]. Postep Derm Alergol 2010; 27: 451-5.

39. Grycewicz J, Cypryk K. Effect of sex hormones on metabolic disturbances in menopausal women [Polish]. Przegl Menopauz 2008; 1: 29-37.

40. Przech E, Cypryk K. Disturbances in glucose metabolism during menopause - clinical implications [Polish]. Nowa Klin 2009; 16: 1115-20

41. Stachowiak G, Stetkiewicz T, Pertyński T. Diabetes mellitus in menopausal women [Polish]. Przegl Menopauz 2011; 3: 173-80.

42. Gottlieb AB, Dann F. Comorbidities in patients with psoriasis. Am J Med 2009; 122: 1150-9.

43. Mallbris L, Grannath F, Hamsten A, et al. Psoriasis is associated with lipid abnormalities at the onset of skin disease. J Am Acad Dermatol 2006; 54: 614-21.

44. SolakTekin N, Tekin IO, Barut F, et al. Accumulation of oxidized low-density lipoprotein in psoriatic skin and changes of plasma lipid levels in psoriatic patients. Mediators Inflamm 2007; 2007: 1-5.
45. Rocha-Pereira P, Santos-Silva A, Rebelo I, et al. Dislipidemia and oxidative stress in mild and severe psoriasis as a risk for cardiovascular disease. Clin Chim Acta 2001; 303: 33-9.

46. Pietrzak A, Michalak-Stoma A, Chodorowska G, et al. Lipid disturbances in psoriasis: an uptade. Mediators Inflamm 2010; 2010.

47. Toruniowa B, Chibowska M, Pietrzak A. Changes in various apolipoprotein levels in psoriasis [Polish]. Przegl Dermatol 1990; 77: 96-101.

48. Preece JF. Raised blood pressure and psoriasis. J R Coll Gen Pract 1997; 27: 713-5.

49. Cohen A, Weitzman D, Dreiher J. Psoriasis and hypertension: a case-control study. Acta Dermatol Venereol 2010; 90: 23-6.

50. Inerot A, Enerback C, Enlund F, et al. Collecting a set of psoriasis family material through a patient organisation: clinical characterisation and presence of additional disorder. BMC Dermatol 2005; 5: 10. 